\title{
Visual Analytics for Data-Driven Analysis in Semiconductor Manufacturing
}

\author{
Patrick Boden*, Sebastian Rank, Thorsten Schmidt ${ }^{1}$ \\ ${ }^{1}$ Chair of Logistics Engineering, Technische Universität Dresden, Münchner Platz 3, 01187 Dresden, Deutschland; \\ *patrick.boden@tu-dresden.de
}

\begin{abstract}
This article dedicates to the process of data visualization. In complex production systems, visualizations are essential to support explorative data analysis and the communication of findings. Quite often, the results of data analyses are static KPIs or visualizations that can only be adapted by extensive preprocessing. This usually requires specific knowledge of software tools that only a few employees have, which hinders an easy adaptation by stakeholders or even a reasonable subsequent use. A new approach called Visual Analytics pretends to overcome the mentioned issues through interactive visualizations combined with automated algorithms.
\end{abstract}

\section{Motivation}

Analysis of system status is crucial for management, control, and optimization of production and logistics systems. For this purpose, industrial applications acquire an increasing amount of data, which must be processed by system experts to generate and communicate information. This process requires the visualization of data because meaningful visualizations support exploratory analysis and enable to share the findings with stakeholders.

Quite often, the results of data analysis are static KPIs, diagrams, or tables. Hence, the value of the analysis is limited and the results can only be used to find answers to initially specified questions. Furthermore, static figures and visualizations are usually not suitable to serve as a basis for communication to stakeholders with different needs like the form of presentation or the selection of the underlying data. Working with un-customized visualizations results in misinterpretations and loss of time due to the time-consuming adaptation of the analysis. Numerous scientific papers about the generation of meaningful visualizations confirm this.

Fortunately, latest approaches intended for exploratory data analysis allow a visual and interaction-based analysis even of a large amount of data. Therefore, sophisticated software tools usually applied in the fields of data management and data analysis are combined with interactive visualizations. They allow the creation of interactive, adaptable dashboards which should allow analysis in depth. The method is also known as Visual Analytics and is the focus of this paper.

The present work summarizes the most important lessons learned from the implementation and application of Visual Analytics applications for visualization tasks in the semiconductor industry.

This work based on the findings gathered in several research projects in the semiconductor industry, as well as literature research in the field of Visual Analytics and own software demonstrators. As the semiconductor domain is characterized by highly complex production systems, new approaches that support data analysis are of particular interest.

\section{State of Technology}

The manufacturing of semiconductor products is highly demanding from a technological and intralogistics point of view. It requires the execution of hundreds of production steps in a cost-intensive production environment to process a wafer which in the end holds the semiconductor chips.

Scientific publications, industry reports, and presentations provide numerous examples for the visualization of data. See for example Ben-Salem et al. (2016), Hammel et al. (2012) and van Roijen et al. (2014).

Usually, results are prepared in form of tables and diagrams. Most common are histograms, pie charts, or box plots. The way of data selection and aggregation, as well as the presentation form rely on the preferences of the author's document which involves the risk of e.g. misinterpretation or wrong focus depending on the addressees' needs.

From our experience, for data analysis and visualization nearly without exception standard software tools are applied. These include for small data sets Excel and for more extensive data sets software tools such as R, Matlab, or Python. Common to all is a static diagram as the result of the visualization process. However, if adaptable graphs are needed, these tools require deep and specific knowledge as source code modifications become necessary.

In general, the following limitations are associated with static reporting:

- $\quad$ Limited access to the data analysis process and the neglect of human capabilities 
- Shortening of information and a lack of adaptability of the analysis

- $\quad$ Specific knowledge of data analysis tools and limited possibilities for collaborative work

Concepts from the field of Visual Analytics could contribute to overcome these limitations.

\section{The Concept of Visual Analytics}

Visual Analytics allows dynamic analysis of data through interactive graphical data exploration combined with automated algorithmic techniques for data analysis. One of the definitions for the term Visual Analytics is provided by Keim et al. (2008): "Visual analytics combines automated analysis techniques with interactive visualizations for an effective understanding, reasoning and decision making on the basis of vast and complex data sets." The approach on the one hand focuses on the strength of computerized analysis, like statistics, data management, and machine learning capabilities. On the other hand, it involves human capabilities such as human cognition or human perception. That allows the analysis of complex data sets in an intuitive way. Patterns found in the analysis can be investigated in several detail levels. New metrics, as well as additional data sets, can enrich the analysis.

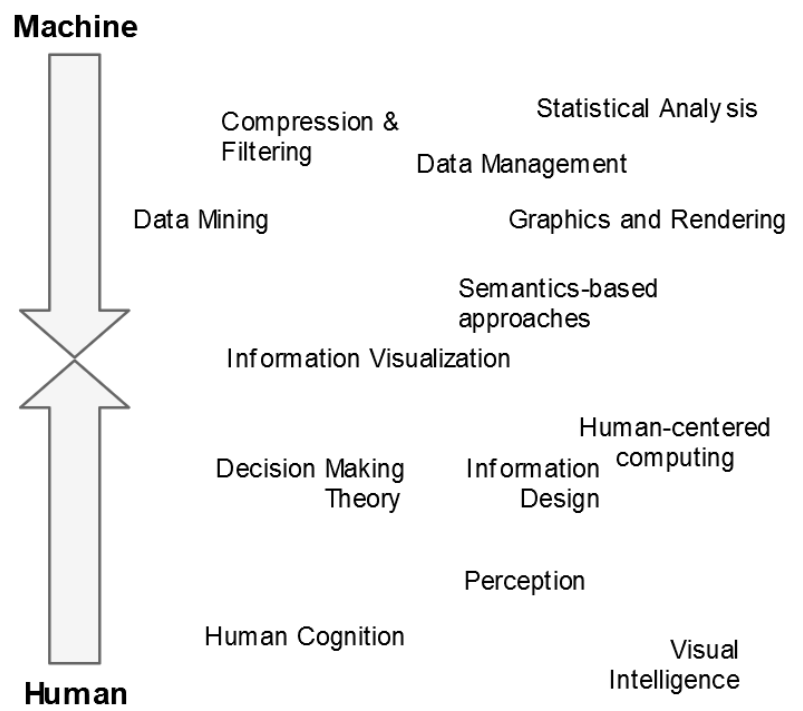

Figure 1: Disciplines integrated into the field of Visual Analytics (see Keim et al. 2008)

An extract of the related disciplines is given in figure 1 . Following Keim et al. (2008) Visual Analytics enables the synthesizing of information from massive data sets, the detection of expected and even unexpected features, and effective communication of findings. For more details about techniques and applications (see Sun et al. 2013).

Visual Analytics requires software tools that allow sophisticated interactive data analysis and visualizations even for large data sets. For this purpose, interaction mechanisms must be provided as well as the automated adaptation of the analysis. Interaction can be enabled in different ways. First, interaction with graphic elements of the visualization is possible by zooming, hovering, or selecting. In this way, parts of the visualization can be examined more closely and areas of interest can be selected for further analysis. Another form of interaction is the selection, rearrangement and expansion of the underlying data. Also, the specific application of statistical methods is an essential way of interaction. Both refers to the use of comparatively simple calculation methods such as averaging up to statistical tests to confirm hypotheses or machine learning applications. The basis for Visual Analytics applications is efficient access to data. In order to achieve the full advantage of Visual Analytics, heterogeneous data from various sources in different levels of aggregation are required and need to be processed in a fast way.

\section{Conclusion}

So far, data analysis in science and industry quite often has been based on static visualizations. Data was highly aggregated and an investigation of addressees' related aspects was nearly impossible.

That is a critical limitation, especially in complex production and logistics systems that depend on various experts' collaborative work. This results in unnecessary delays due to time-consuming adaptation of the analysis and severely limited access to and understanding of the data.

Software tools from the area of Visual Analytics offer intuitive access to the analysis of the data. Dashboards prepared by experts can be adapted and extended even with limited knowledge of the respective domain. By combining human strengths with automated data processing, new insights can be gained.

\section{References}

[1] Ben-Salem, A.; Yugma, C.; Troncet, E.; Pinaton, J.: AMHS design for reticles in photolithography area of an existing wafer fab. In: 27th Annual SEMI Advanced 
Semiconductor Manufacturing Conference (ASMC), 2016, pp. 110-115.

[2] Hammel, C.; Schmidt, T.; Schöps, M.: Network optimization prior to dynamic simulation of AMHS. In: Proceedings of the 2012 Winter Simulation Conference, 2012, pp. 1956-1966.

[3] Keim, D.; Andrienko, G.; Kekete, J.-D.; Görg, C.; Kohlhammer, J.; Melancon, G.: Visual Analytics: Definition, Process, and Challenges. In: Information Visualization, 2008, pp. 154-175.

[4] Sun, G.-D.; Liang, R.-H.; Liu, S.-X.: A Survey of Visual Analytics Techniques and Applications: State-of-theArt Research and Future Challenges. In: Journal of computer science and technology, 2013, pp. 852-867.

[5] Van Roijen, R.; Joshi, P.; Bailey, D.; Conti, S.; Brennan, W.; Findeis, P.: Defect reduction by nitrogen purge of wafer carriers. In: ASMC 2013 SEMI Advanced Semiconductor Manufacturing Conference, 2013, pp. 338341.

\section{Note}

The project iDev40 has received funding from the ECSEL Joint Undertaking under grant agreement No. 783163. The JU receives support from the European Union's Horizon 2020 re-search and innovation program. It is co-funded by the consortium members, grants from Austria, Germany, Belgium, Italy, Spain and Romania. It is coordinated by Infineon Technologies Austria AG.

The information and results set out in this publication are those of the authors and do not necessarily reflect the opinion of the ECSEL Joint Undertaking.
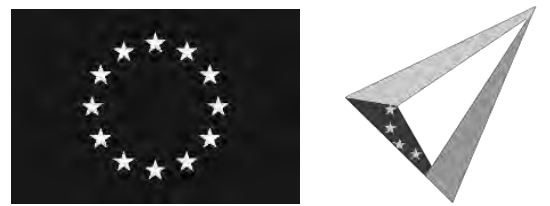